\title{
Effects of Biotic Interactions, Abiotic Environments and Harvesting on the Spread of Hantavirus Infection
}

\author{
${ }^{1}$ F. M. Yusof, ${ }^{2}$ M. H. Mohd*, ${ }^{3}$ Y. Mohd Yatim and ${ }^{4}$ A. I. Md. Ismail \\ ${ }^{1}$ Faculty of Science and Mathematics, Sultan Idris Education University \\ 35900 Tanjong Malim, Perak, Malaysia \\ ${ }^{2,3,4}$ School of Mathematical Sciences, Universiti Sains Malaysia \\ 11800 USM, Pulau Pinang, Malaysia \\ ${ }^{*}$ Corresponding author: fauzi.my@fsmt.upsi.edu.my
}

Article history

Received: 17 December 2018

Received in revised form: 22 September 2019

Accepted: 10 December 2019

Published online: 1 April 2020

\begin{abstract}
In this paper, the combined influences of biotic interactions, environmental components and harvesting strategy on the spread of Hantavirus are investigated. By employing a multi-species model consisting of (susceptible and infected) rodents and alien species, we show that interspecific competition from alien species has an effect in reducing the spread of infection, and this species could be employed as a potential biocontrol agent. Our analysis using numerical continuation and simulation also reveals the conditions under which Hantavirus infection occurs and disappears as the environmental conditions and the intensity of harvesting change. Without harvesting, infection emerges when environments are conducive. Inclusion of moderate harvesting in favourable environments can lead to disappearance of infection among rodent species. However, as the intensity of harvesting increases, this situation can cause extinction of all rodents species and consequently, jeopardise biodiversity. Overall, our results demonstrate how the interplay of different factors can combine to determine the spread of infectious diseases.
\end{abstract}

Keywords Hantavirus; competition model; biotic interactions; abiotic environments; harvesting; biodiversity model

Mathematics Subject Classification 92B05

\section{Introduction}

Hantaviruses are tri-segmented negative sense, single-stranded RNA viruses spread by specific rodents [1] This virus can cause Hantavirus pulmonary syndrome (HPS) and Hantavirus renal syndrome (HFRS) which can result in deaths. The disease is transmitted by infected rodents through excreta, saliva or urine. Recently, a new Hantavirus has been found in shrews, moles and bats from Africa and Asia. This virus, named Makokou virus (MAKV), is hosted by bats and is an important reservoir for emerging human pathogens [2]. 
A mathematical model to describe the basic features of the spread of Hantavirus infection was developed by Abramson and Kenkre [3]. The construction of the basic model of Abramson and Kenkre [3] (AK model) incorporates decay by death of the mice population, the increase by birth and effect of the environment to stabilize the population [4]. The AK model is able to successfully explain field observations such as environmentally controlled phase transitions, thus providing an analytical support to biological hypotheses such as the trophic cascade [5]. Several extensions to this basic model have been developed by Abramson et al. [6], Kenkre et al. [7], Abdul Karim et al. [8], Goh et al. [4], Yusof et al. [9]. Rida et al. [10] and Yusof et al. $[11-13]$.

An uncontrolled increase in rodent population causes Hantavirus infection to spread and so there is a need to limit the population of rodents. One possible way is to implement population harvesting. According to Hale and McCarthy [14], harvesting is defined as reduction of the population due to hunting or capturing individuals, which in effect removes individuals from the population. Such a policy has been used to stabilise population in an environment with limited resources or environmental parameter. Kar and Pahari [15] stated that harvesting has a strong bearing on the dynamic evolution of a population. The long-run stationary density of the population can be significantly smaller than the long-run stationary density of a population in the absence of harvesting, depending on the nature of the applied harvesting strategy. It was pointed out that a population can be free of extinction risk in the absence of harvesting. Furthermore, harvesting may lead to the incorporation of a positive extinction probability and to potential extinction in finite time.

Solomon et al. [16] states that biodiversity is decreasing worldwide. Ecosystem biodiversity is one aspect of biodiversity. In real-life, rodents not only share resources among themselves, but they also share with other species (so called alien species). The competition between rodents and alien species should be taken into account.

Some researches that have been conducted indicate that biodiversity plays an important role to control the spread of Hantavirus [17-18] Peixoto and Abramson [18] studied the effect of biodiversity on the prevalence of the infection using a mathematical model in which a single alien population competes with the host i.e., rodents. In general, a particular Hantavirus has a specific host which remains infected and the infection is maintained by horizontal transmission, i.e., it does not pass on the disease to its Offspring (called vertical transmission). In the present paper, we explore the effects of biotic interactions, environmental components and population harvesting on the spread of Hantavirus infection. The effects of varying parameters of interest are investigated in details using numerical continuation and numerical simulation. For numerical continuation, we use XPPAUT software [19] and for numerical simulation, we use MATLAB.

\section{The Model}

We employ a multi-species model by extending the community model of Peixoto and Abramson [18] to include the effects of population harvesting [9]. Specifically, we consider a system of ordinary-differential equations (ODE) for the densities of susceptible rodent $\left(r_{S}\right)$, infected rodent $\left(r_{i}\right)$ and alien species $\left(z_{a}\right)$ : 


$$
\left.\begin{array}{l}
\frac{d r_{s}}{d t}=b r-c r_{s}-\frac{r_{s}}{k}\left(r+q z_{a}\right)-a r_{s} r_{i}-E r_{s}, \\
\frac{d r_{i}}{d t}=-c r_{i}-\frac{r_{i}}{k}\left(r+q z_{a}\right)+a r_{s} r_{i}-E r_{i}, \\
\frac{d z_{a}}{d t}=(\beta-\gamma) z_{a}-\frac{z_{a}}{k}\left(z_{a}+\varepsilon r\right) .
\end{array}\right\}
$$

where $r$ is the total population of rodents, $a$ is the horizontal transmission rate of Hantavirus, $b$ is the birth rate of rodent species, $c$ is the death rate of rodent species, $\beta$ is the birth rate of alien species, $\gamma$ is the death rate of alien species, $q$ is the competitive effect of alien species on rodents, $\varepsilon$ is the competitive effect of rodents on alien species, $k$ is carrying capacity and $E$ is the harvesting efforts. Consistent with previous studies $[3,18]$, we model the suitability of environments or habitats using parameter $k$ and we assume that all the species have equal carrying capacity. These habitats can vary in environmental conditions or availability of resources, such as water and food. Thus, higher values of $k$ indicate a favourable environment with higher availability of resources; this situation can cause species to thrive.

\section{Analysis}

We investigated the dynamical behaviours of the model by varying the strength of biotic interactions $(q$ and $\varepsilon$ ), environmental carrying capacity $(k)$ and harvesting effort $(E)$ using numerical continuation. We first ignored the influence of harvesting (i.e. setting $E=0$ ) and examined how interspecific competition determines the community dynamics. To summarize the outcomes of species interactions, we constructed parameter space in Figure 1 by varying the competition coefficients $q$ and $\varepsilon$. We divided the $q-\varepsilon$ plane into regions of qualitatively different dynamics, identifying those regions in parameter space where species coexistence, species exclusion and priority effects occur. Transcritical bifurcations occur as one crosses the boundaries of these regions, by which two steady states exchange their stabilities. We then explored the combined effects of environmental factor and harvesting on Hantavirus infection by letting the parameters $k$ and $E$ to change in our model. Similar to the previous analysis, we also constructed a co-dimension two bifurcation diagram (Figure 4) and identified different regions in $E-k$ space where infection (dis-)appear or lead to biodiversity loss. For numerical continuation and bifurcation analysis, we employed XPPAUT: Equation (1) was solved for steady state using cvode solver for $t=100$. Then, the steady state was tracked in AUTO, in which we tracked stable and unstable steady states and also bifurcation points as model parameters change.

By performing invasion analysis, we found most curves that divide the $q-\varepsilon$ (grey lines) and $E$ - $k$ (black curves). These curves correspond to the threshold values for biologically-relevant parameters (e.g. $k_{C}, k_{H}, E_{C}, q *, \varepsilon *$ ) when the dynamical behaviours of the system change. This analysis was conducted by determining when the per capita growth rate of a rare species (the invader) was positive or negative under the conditions set by the other species. Using MAPLE,

we found exact analytical expressions for all curves in Figure 1 and Figure 4. So, the positive equilibrium points of the Equation (1) are

$$
E_{0}(0,0, k(\beta-\gamma)), E_{1}\left(\frac{b}{a},-\frac{a k(E-b+c)+b}{a}, 0\right),
$$




$$
E_{2}\left(\frac{k((E-b+c)+q(\beta-\gamma))}{q \varepsilon-1}, 0-\frac{k(\varepsilon(E-b+c)+(\beta-\gamma))}{q \varepsilon-1}\right) \text { and } E_{3}\left(r_{s} *, r_{i} *, z_{a} *\right),
$$

where

$$
r_{s} *=\frac{b}{a}, \quad r_{i} *=\frac{a k(q(\beta-\gamma)-(b-c-E))-b(\varepsilon q-b)}{a(q \varepsilon-1)}
$$

and

$$
z_{a} *=-\frac{k \varepsilon(E-b+c)+k(\beta-\gamma)}{q \varepsilon-1}
$$

Overall, comparisons of analytical with numerical continuation results on these thresholds have indicated agreement between the two findings.

To better understand what happens in each region of our parameter space diagrams, we also presented several numerical simulation results (abundance vs. time plots) using different sets of parameter values (Figures 2, 3, 5-7). For numerical simulation results, Equation (1) was solved numerically using Runge-Kutta method with MATLAB ode45 solver for $t=100$. We used initial conditions as indicated in each figure section. To verify that the steady state was stable, Equation (1) was analysed using MAPLE linalg package by seeking steady states and performing a stability analysis. The steady state was stable if all eigenvalues have negative real parts. Unless otherwise stated, parameter values used in this paper were given in Table 1, which were based on empirical studies by Peixoto and Abramson [18]

Table 1: Parameter Values

\begin{tabular}{|c|l|c|}
\hline Symbol & \multicolumn{1}{|c|}{ Description } & Parameter Value \\
\hline$a$ & The transmission rate of Hantavirus & 0.1 \\
\hline$b$ & The birth rate of rodent species & 1 \\
\hline$c$ & The death rate of rodent species & 0.6 \\
\hline$\beta$ & The birth rate of alien species & 1 \\
\hline$\gamma$ & The death rate of alien species & 0.5 \\
\hline$q$ & Competitive effect of alien species on rodents & Varied \\
\hline$\varepsilon$ & Competitive effect of rodents on alien species & Varied \\
\hline$k$ & Carrying capacity of environment & Varied \\
\hline$E$ & Harvesting efforts & Varied \\
\hline
\end{tabular}

\section{Results}

\subsection{The Effects of Biotic Interactions on the Spread of Hantavirus infection}

In this section, we explore the effects of biotic interactions on rodents $\left(r_{s}\right.$ and $\left.r_{i}\right)$ and alien $\left(z_{a}\right)$ species. Without harvesting $(E=0)$, the dynamics of the model depend on the strength of biotic interactions (i.e. competition). For example, Figure 1 shows the population dynamics in favourable environment $(k=150)$ as the competitive strength of rodents $(\varepsilon)$ and alien $(q)$ change in the system. This plot was generated using two different initial conditions: initial abundances favour rodent populations $\left(r_{s}\right.$ and $\left.r_{i}\right)$ and initial abundance favours alien species $\left(z_{a}\right)$. Different colour regions represent different combinations of species presences, with the 


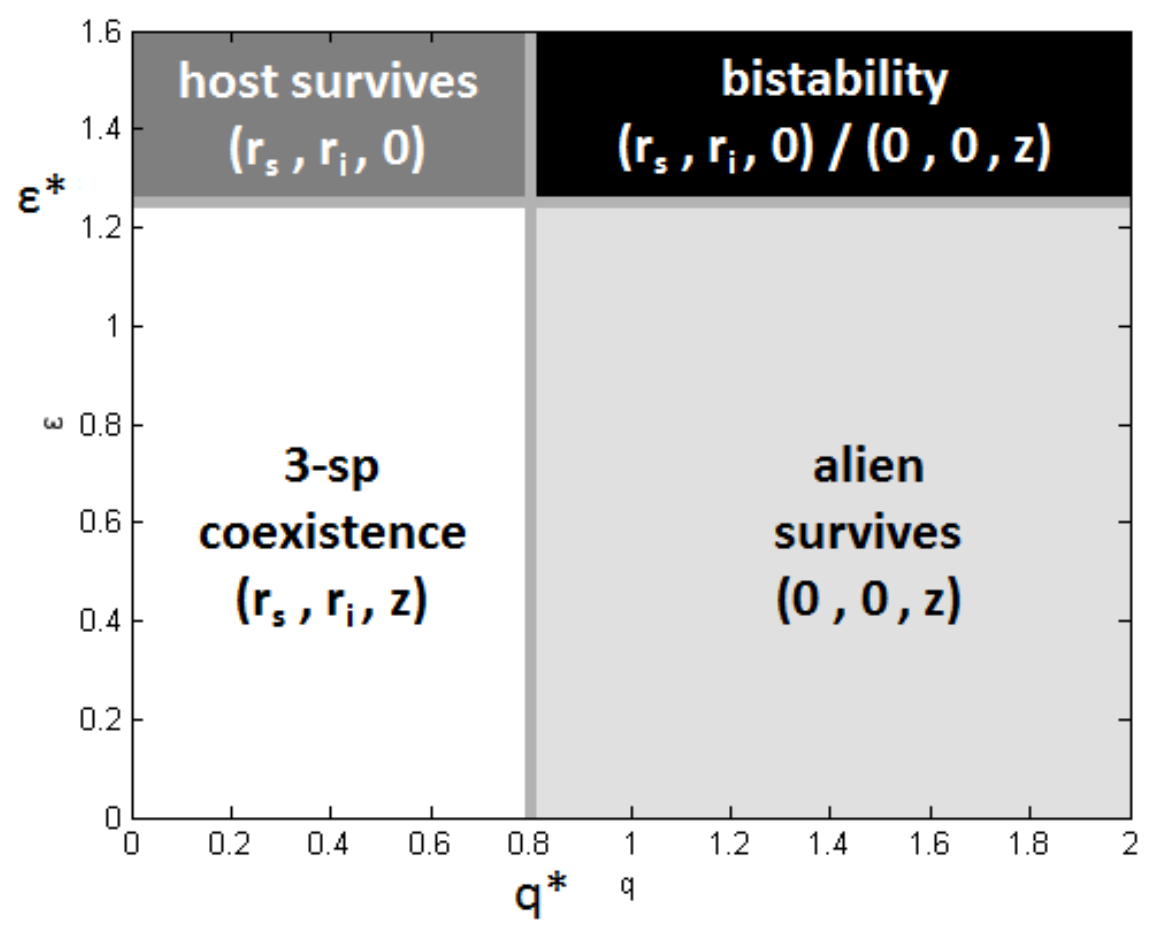

Figure 1: Bifurcation Diagram of the Multi-species Model (1) without Harvesting $(E=0)$ Showing the Effects of Biotic Interactions.

boundaries between these regions (grey lines) corresponded to the occurrence of transcritical bifurcations in the model. These bifurcations indicate the threshold values in competitive strength $q *$ and $\varepsilon *$, which can result in qualitatively different dynamics. The analytical expressions for these thresholds were given by:

$$
q *=(c-b) /(\gamma-\beta) \text { and } \varepsilon *=(\gamma-\beta) /(c-b) \text {. }
$$

When competition was relatively weak $(q<q *, \varepsilon<\varepsilon *)$, multi-species coexistence was possible (white region). In this situation, the susceptible and infected rodents could coexist with the alien species (Figure 2a). When either one of these species exerted strong biotic interactions $(q>q *, \varepsilon<\varepsilon *$ or $q<q *, \varepsilon>\varepsilon *)$, exclusion of species occured due to competitive asymmetry (light and dark grey regions). This is illustrated in Figure 2(b), where, due to strong asymmetriccompetition of rodents on alien species $(q<q *, \varepsilon>\varepsilon *)$, only rodent species could survive and the alien species was displaced. Similar graphs could be plotted for the case $(q>q *, \varepsilon<\varepsilon *)$.

From an ecological viewpoint, this case corresponds to Gause's competitive exclusion principle [20], in which only the strongest species can survive and that coexistence is impossible. When all species exert strong interspecific competition $(q>q *$ and $\varepsilon>\varepsilon *)$, there can be alternative stable states. Ecologically, this is known as priority effects $([21,22])$. In this situation, the system shows bistable behaviour (black region), with two different steady states: either the rodent populations (Figure 3(a)) or alien (Figure 3(b)) can survive. Which steady state is reached depends on the initial abundances of species. 


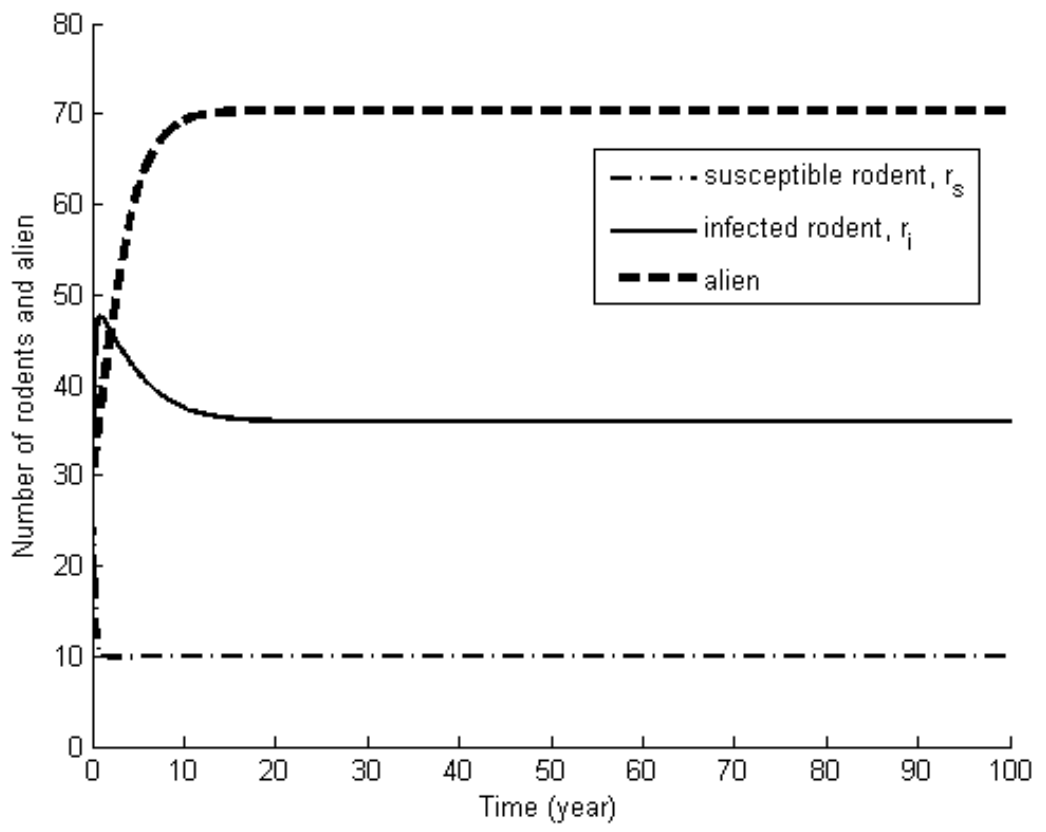

(a)

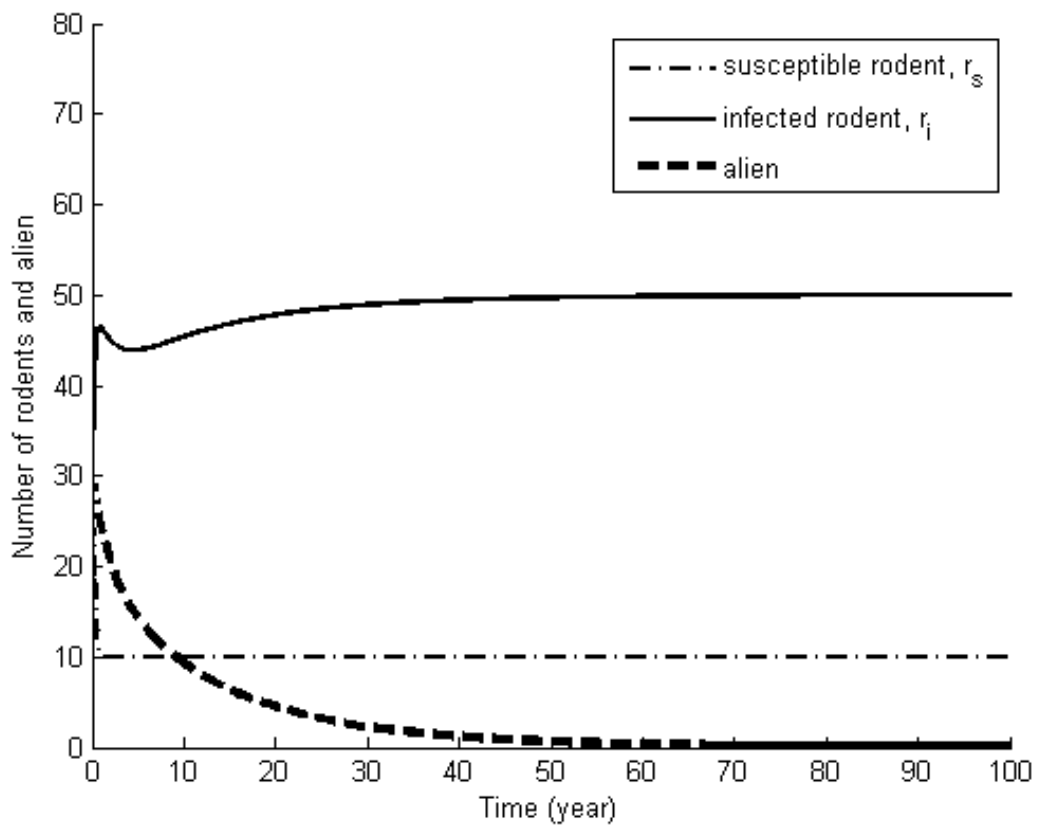

(b)

Figure 2: Population of $r_{s}, r_{i}$ and $z_{a}$ for Multi-species Model without Harvesting $(E=0)$ with Initial Values $r_{s}=r_{i}=z_{a}=30$, for a) $q<q *, \varepsilon<\varepsilon *$ and b) $q<q *, \varepsilon>\varepsilon *$. 


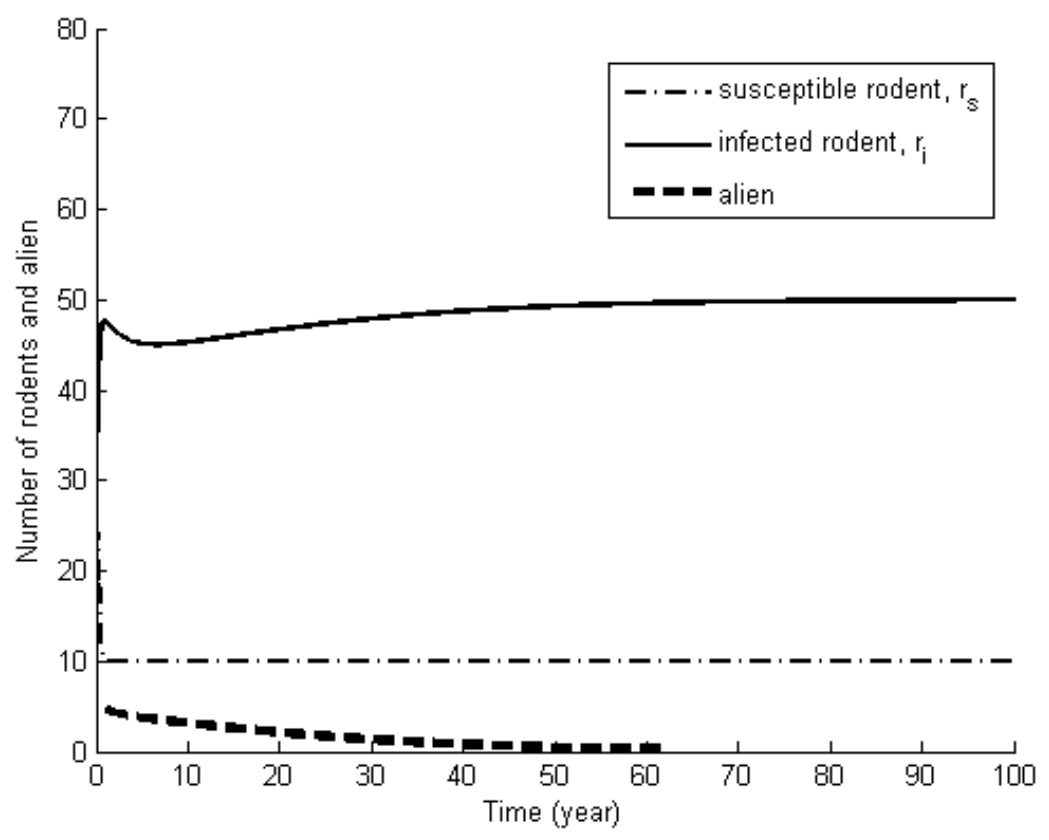

(a)

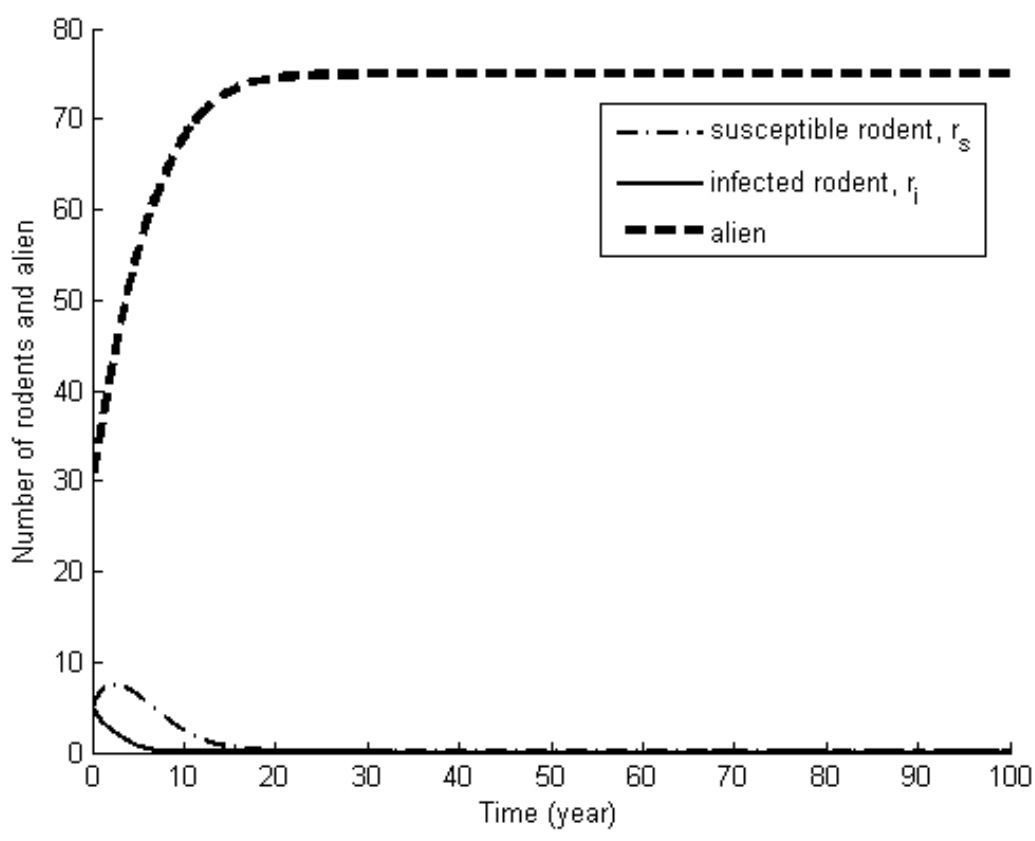

(b)

Figure 3: Population of $r_{s}, r_{i}$ and $z_{a}$ for Multi-species Model without Harvesting $(E=0)$ and in the Presence of Strong Inter Specific Competition $(q>q *$ and $\varepsilon>\varepsilon *)$, with (a) Initial Abundances Favour Rodent Species $\left(r_{s}=30, r_{i}=30, z_{a}=5\right)$, and (b) Initial Abundances Favour Alien Species $\left(r_{s}=5, r_{i}=5, z_{a}=30\right)$. 
Based on these findings, biotic interactions can have different effects on the outcome of species interactions: on one hand, relatively weak biotic interactions permit coexistence of multiple species; on the other hand, strong biotic interactions can result in exclusion of species. Consequently, either rodent or alien species can survive due to competitive asymmetry or priority effects. We observe that Hantavirus infection in rodent populations disappears $\left(r_{i}=0\right)$ when interspecific competition exerted by the alien species $\left(z_{a}\right)$ is relatively strong $(q>q *)$. However, this situation leads to extinction of susceptible rodent $\left(r_{s}\right)$ as well, which can result in the loss of biodiversity. To overcome this problem, in the next section, we consider the joint influences of environmental factors and harvesting in controlling the spread of Hantavirus infection.

\section{The Joint Effects of Abiotic Environments and Harvesting on the Spread of Hantavirus Infection}

We investigate the combined influences of abiotic environments and harvesting by varying the carrying capacity $(k)$ and harvesting effort $(E)$, respectively. To emphasise parallel with previous empirical studies [18], we consider the case when biotic interactions are relatively weak $(q<q *, \varepsilon<\varepsilon *)$.

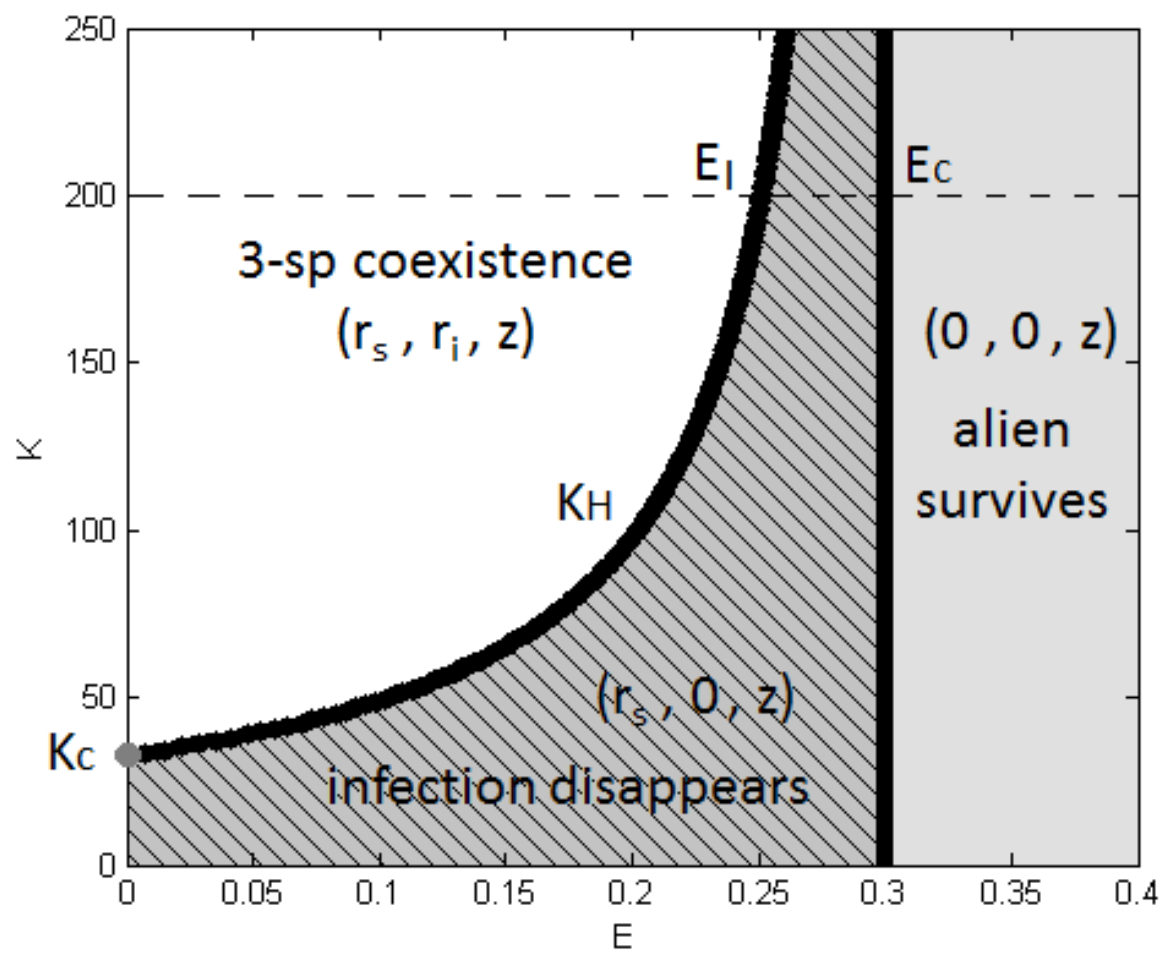

Figure 4: Bifurcation Diagram of the Multi-species Model (1) Showing the Effects of Environmental Factors and Harvesting Strategy

For instance, Figure 4 shows community composition as parameters $E$ and $k$ are varied. In general, $k$ represents the suitability of environments/habitats. In the absence of harvesting $(E=0)$, there occurs a critical value for carrying capacity $\left(k_{c}\right)$, given by the following 
expression:

$$
k_{C}=\frac{b(q \varepsilon-1)}{a(q \beta-q \gamma-b+c)} .
$$

The threshold $k_{c}$ corresponds to a transcritical bifurcation point (Figure 4: grey point); for $k>k_{c}$ (i.e. favourable environments), the density $r_{i}$ tends toward positive steady state $\left(r_{i}>0\right)$ and thus infection persists (Figure 5(a)); for carrying capacity $k<k_{c}$ (adverse environments), infection vanishes $\left(r_{i}=0\right)$ and only susceptible rodent and alien species presence (Figure $5(\mathrm{~b})$ ).

When harvesting strategy is considered, we observe the conditions under which a trade-off between harvesting and environmental effects can lead to (dis-)appearance of infection. In this case, the parameter space (Figure 4) is divided into several regions of qualitatively different dynamics: three-species coexistence (white region), infection vanishes (grey shaded region) and only alien survives (grey region). Inclusion of harvesting strategy $(E>0)$ can result in the threshold for environmental conditions to be shifted to higher values of $k$. It is now given by the following expression:

$$
k_{H}=\frac{b(q \varepsilon-1)}{a(q \beta-q \gamma+E-b+c)} .
$$

In the $k-E$ plane (Figure 4 ), the threshold $k_{H}$ corresponds to transcritical bifurcation (black curve). When $E>0$, this strategy has the effect of reducing the prevalence of infection in favourable environments. For example, in conducive environments (e.g. $k=200$, dotted line), infection emerges when $E<E_{I}$ (white) and consequently, multi-species steady state (Figure 6a) is stable. When $E>E_{I}$ (grey shaded), Hantavirus infection disappears, and this situation leads to the survival of susceptible rodent and alien species (Figure 6b).

We also observe the threshold value for harvesting strategy $E_{C}$ beyond which extinction of all rodent populations occur and only alien species can survive. This situation occurs when the denominator of Equation (4) becomes very small $(q \beta-q \gamma+E-b+c \rightarrow 0)$, and the threshold $E_{C}$ is given by the following expression:

$$
E_{C}=b-c+q(\gamma-\beta)
$$

The threshold $E_{C}$ corresponds to the extreme harvesting efforts, which can jeopardise our biodiversity and lead to extinction of all rodent species. This is illustrated in Figure 7 where, due to harvesting in an unsustainable manner, only alien species survives and rodent species go extinct. Based on these results, the optimal harvesting strategy is achieved when $E_{I}<E<E_{C}$

(grey shaded region). In this situation, the prevalence of infection is completely eliminated and the maintenance of susceptible rodent $\left(r_{S}\right)$ and alien species $\left(z_{a}\right)$ is possible.

\section{Discussion and Conclusion}

In this paper, we employed a multi-species model to investigate the combined influences of biotic interactions, abiotic environments and harvesting strategy on the occurrence of Hantavirus infection. We find that both biotic and abiotic forces determine community dynamics of (susceptible and infected) rodents and alien species. Our results also show that harvesting strategy can have different effects on species diversity and the prevalence of infection: while 


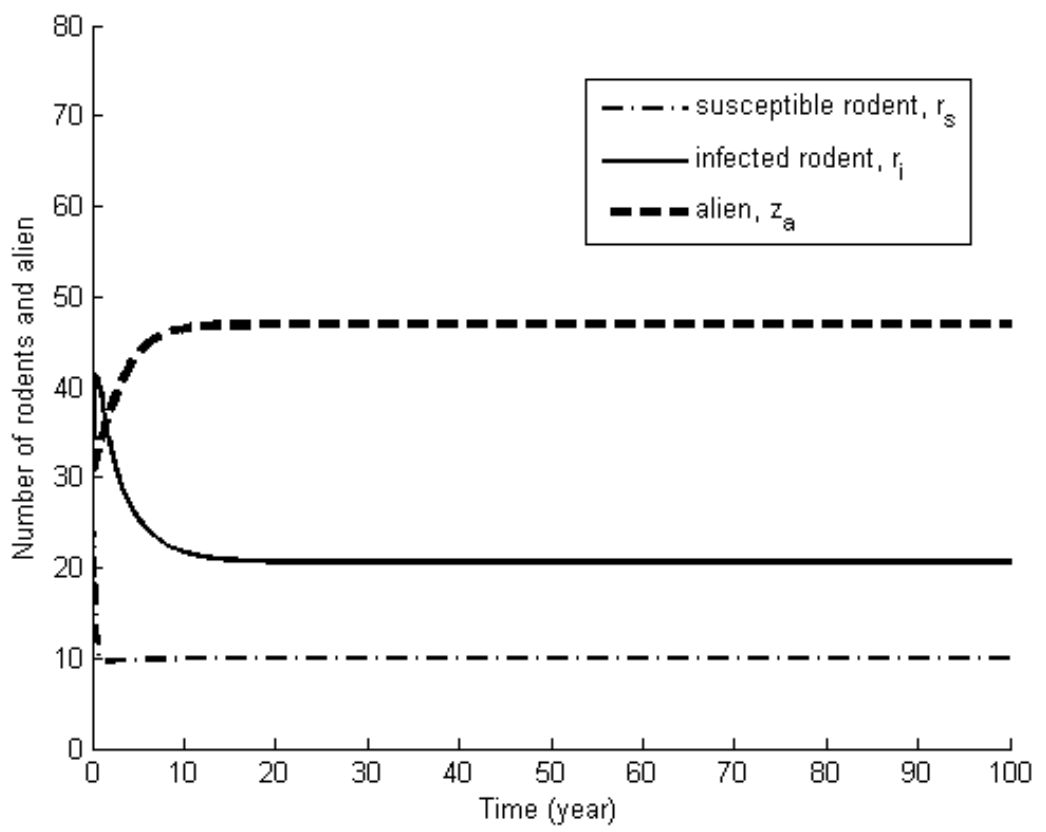

(a)

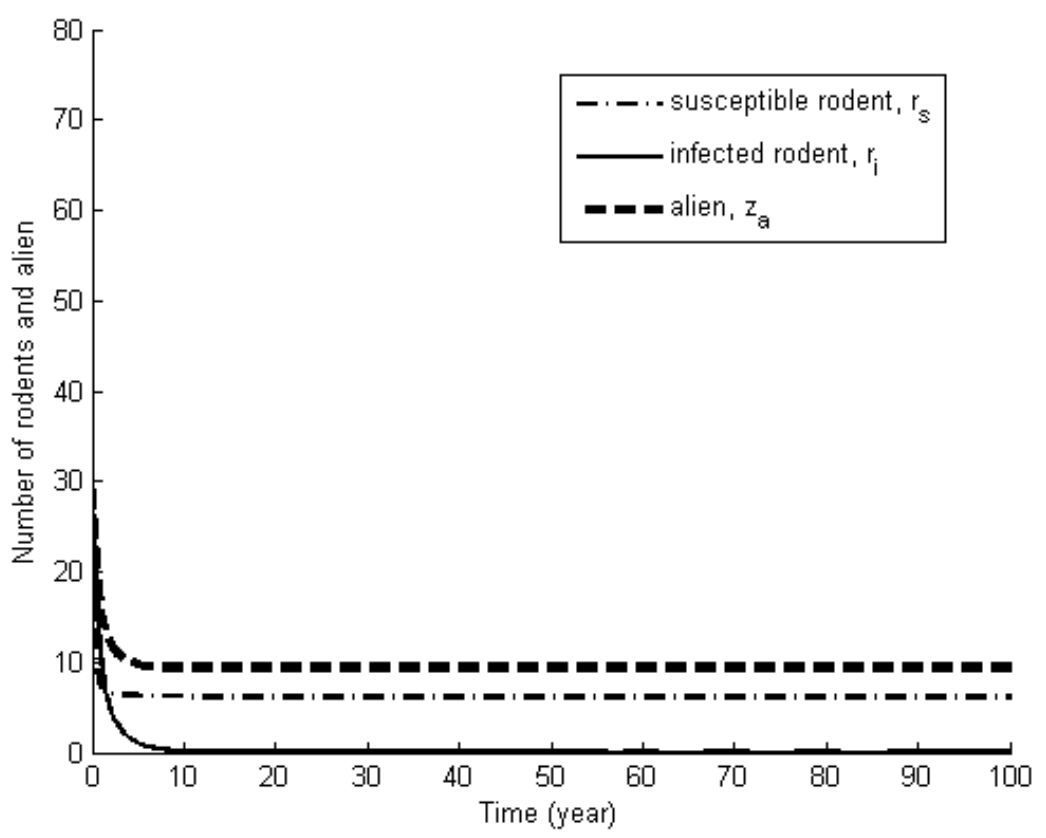

(b)

Figure 5: Population of $r_{s}, r_{i}$ and $z_{a}$ for Multi-species Model without Harvesting $(E=0)$ and Under Variation in Environmental Conditions $(k)$ : (a) $k=100\left(k>k_{c}\right)$; (b) $k=20\left(k<k_{c}\right)$. These Plots are Generated using Initial Abundances $r_{s}=r_{i}=z_{a}=30$. 


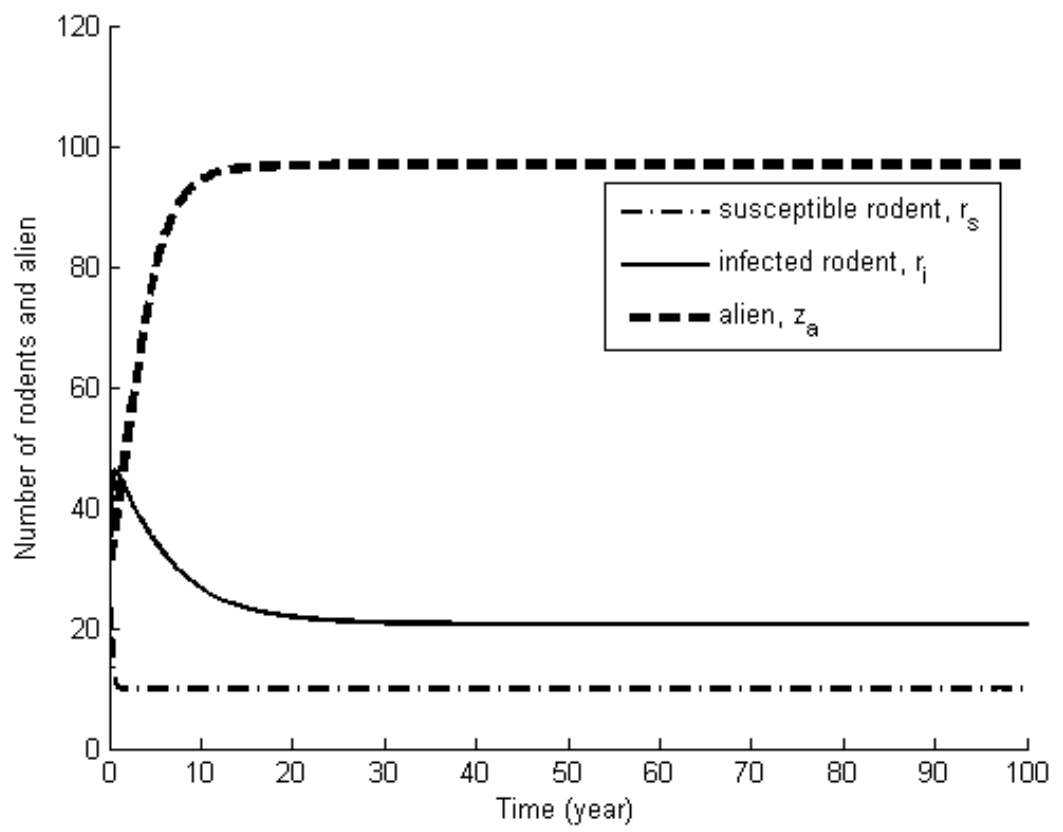

(a)

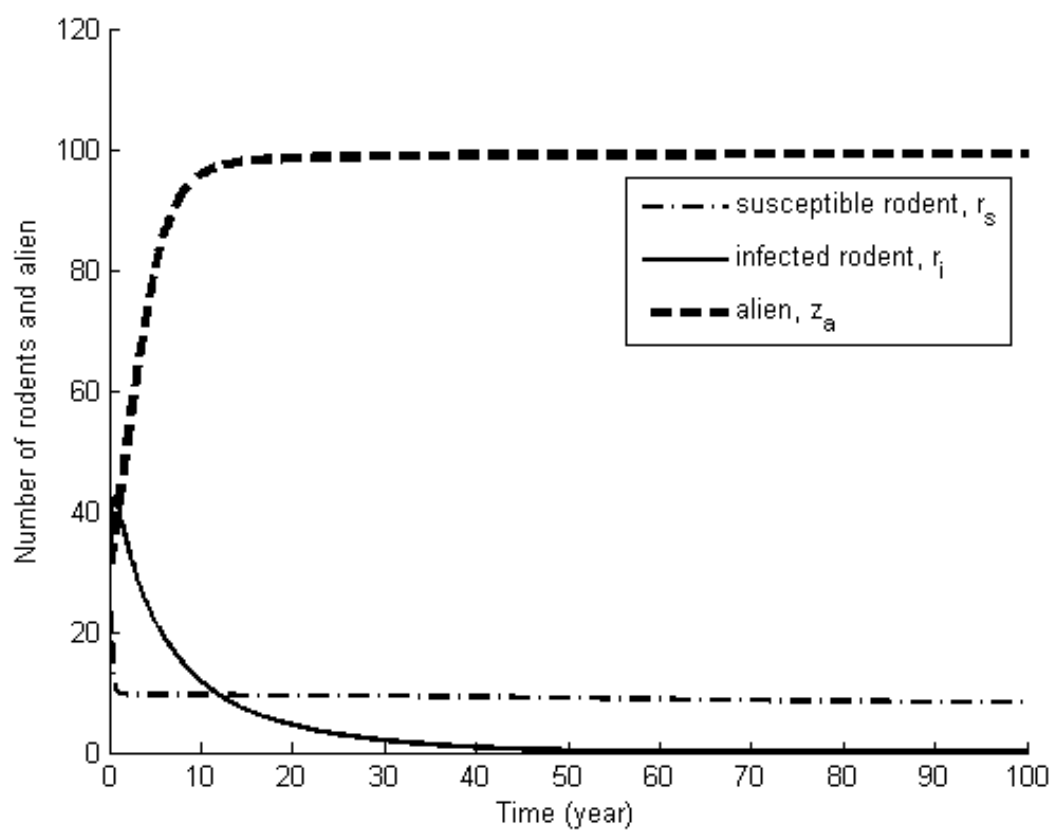

(b)

Figure 6: Population of $r_{s}, r_{i}$ and $z_{a}$ for Multi-species Model with Harvesting $(E>0)$ and under Favourable Environmental Conditions (e.g. $k=200)$ : (a) $E=0.15\left(E<E_{I}\right)$; b) $E=0.25$ $\left(E>E_{I}\right)$. These Plots are Generated using Initial Abundances $r_{s}=r_{i}=z_{a}=30$. 


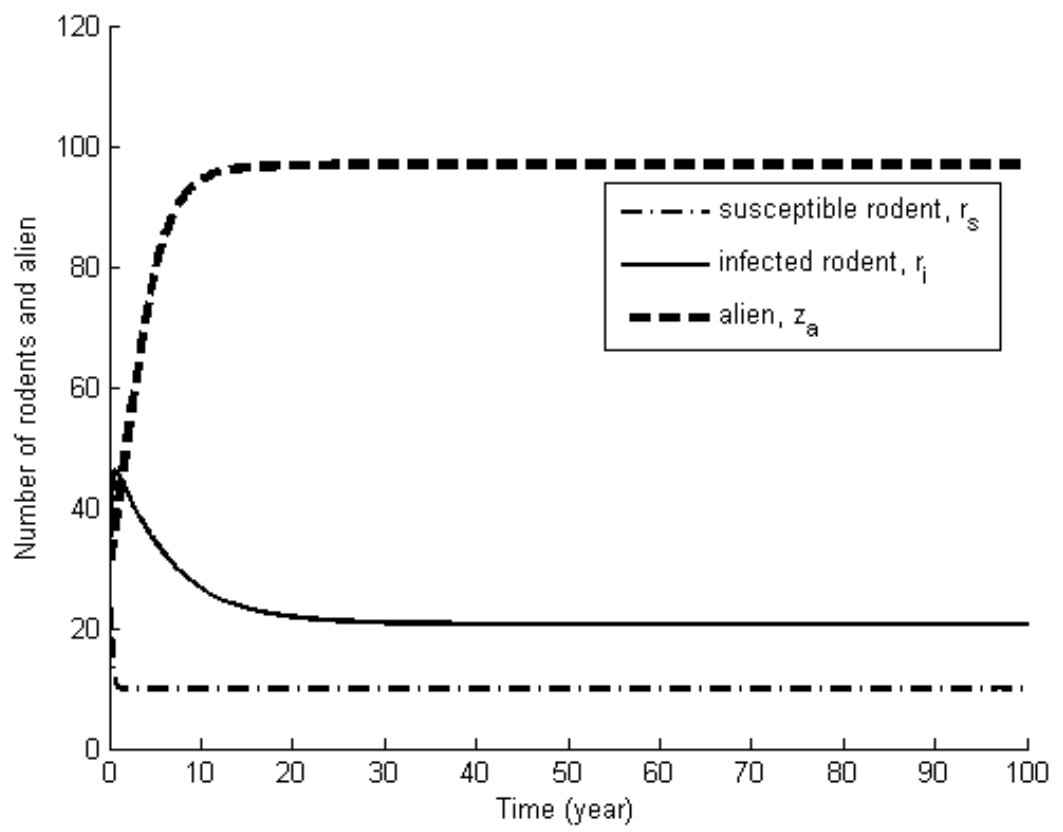

(a)

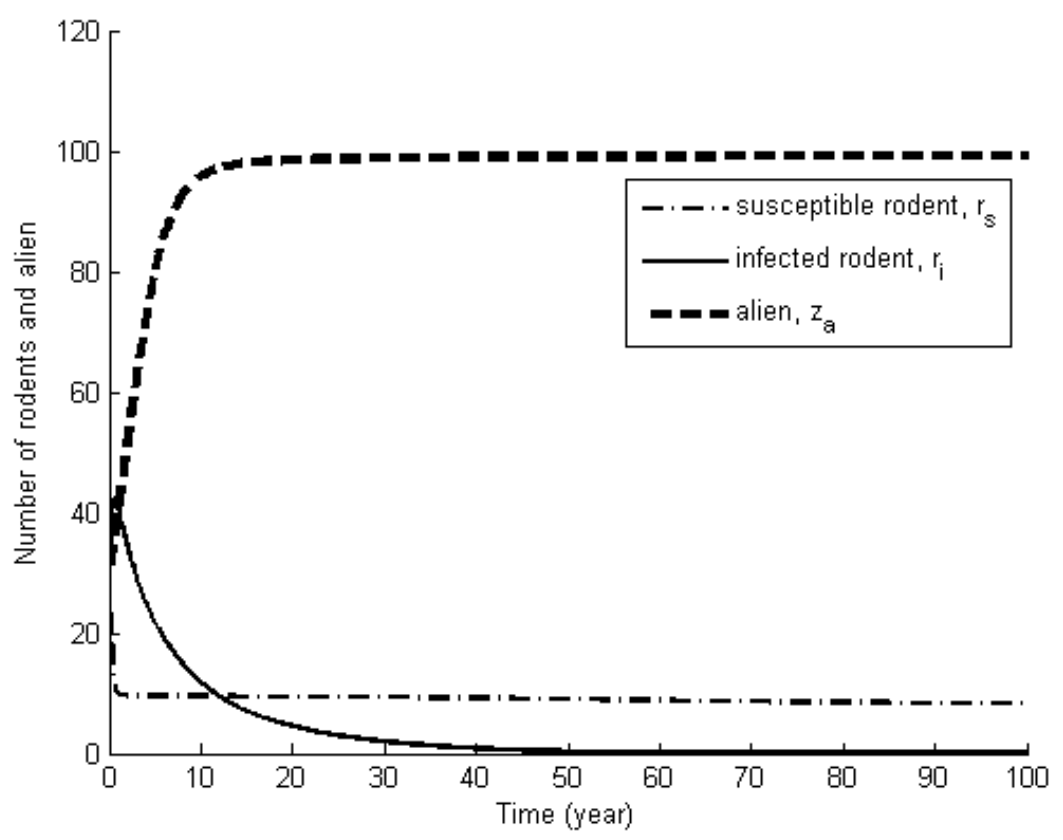

(b)

Figure 7: Population of $r_{s}, r_{i}$ and $z_{a}$ for Multi-species Model with Extreme Harvesting Efforts $\left(E>E_{C}\right)$ and under Two Environmental Conditions: (a) $k=20$ (Adverse Environments); (b) $k=200$ (Favourable Environments). These Plots are Generated using $E=0.35$ and Initial Abundances $r_{s}=r_{i}=z_{a}=30$. 
moderate harvesting efforts can lead to disappearance of infection, overharvesting can cause biodiversity loss and consequently only alien species can survive.

We also derived several analytical expressions, which represent the threshold values for competitive strength, carrying capacity and harvesting efforts beyond which Hantavirus infection (dis-)appear. By employing continuation methods, we show that these threshold values correspond to transcritical bifurcations of dynamical systems. For instance, Equation (3) gives an expression of the critical value for environmental parameter $\left(k_{c}\right)$ when harvesting is excluded $(E=0)$. This finding is in line with some theoretical and empirical evidence $[2,14]$ : these studies illustrate the occurrence of threshold environmental parameter $k$ above which the population shows a positive prevalence of infection. We also demonstrate that the threshold value for environmental parameter is shifted to higher $k$-values when harvesting is incorporated $\left(k_{H}\right.$, given by Equation (4)).

Our results may have some qualitative implications on the use of harvesting in controlling the spread of Hantavirus. We obtain an analytical expression for over harvesting activity $\left(E_{C}\right.$, given by Equation (5)), which can provide precautionary measures in controlling Hantavirus infection and species diversity. In general, the use of harvesting strategy to prevent the spread of Hantavirus should be employed with caution. This is because extreme harvesting strategy $\left(E>E_{C}\right)$ can lead to extinction of vulnerable rodent species $\left(r_{S}\right)$ and jeopardise our biodiversity. Thus, to ensure optimal harvesting strategy, this may require accurate information on biotic interactions, as well as knowledge on abiotic environments.

In sum, the community model that we examined help us to better understand how the interplay of different ecological forces (e.g. biotic and abiotic components) and external factor (e.g. harvesting) can combine to result in (dis-)appearance of Hantavirus infection. It is hope that the insights from our model will open the door toward further research in epidemiology and other exploration for the control of Hantavirus infection.

\section{References}

[1] Vaheri, A., Henttonen, H. Voutilainen, L., Mustonen, J., Sironen, T. and Vapalahti, O. Hantavirus infections in Europe and their impact on public health. Rev. Med. Virol. 2013. 23: $35-49$.

[2] Witkowski, P. T., Drexler, J. F., Kallies R., Liková M., Bokorová, S., Gael D., Mananga G. D., Szemes T., Leroy, E. M. , Krüger, D. H., Drosten, C. and Klempa, B. Phylogenetic analysis of a newfound bat-borne Hantavirus supports a laurasiatherian host association for ancestral mammalian Hantaviruses. Genet. Evol. 2016. 41: 113-119.

[3] Abramson, G. and Kenkre, V. M. Spatiotemporal patterns in the Hantavirus infection. Physical Review E 66. 2002. 011912-1-5.

[4] Goh, S. M., Ismail, A. I. M., Noorani, M. S. M. and Hashim, I. Dynamics of the Hantavirus infection through variational iteration method (VIM). Nonlinear Analysis: Real World Applications. 2009. 10(4): 2171-2176.

[5] Abramson, G. Mathematical modeling of Hantavirus: from the mean field to the individual level. In: Progress in Mathematical Biology Research Editor: James T. Kelly. 2007. 1-27. 
[6] Abramson, G., Kenkre, V. M., Yates, T. L. and Parmenter, B. R. Traveling waves of infection in the Hantavirus epidemics. Bulletin of Mathematical Biology. 2003. 65(3): 519534.

[7] Kenkre, V. M., Giuggioli, L., Abramson, G. and Camelo-Neto, G. Theory of Hantavirus infection spread incorporating localized adult and itinerant juvenile mice. Eur. Phys. J. B. 2006. 55(4): 461-470.

[8] Abdul Karim, M. F., Ismail, A. I. M. and Ching, H. B. Cellular automata modeling of Hantavirus infection. Chaos, Solitons \& Fractals. 2009. 41(5): 2847-2853.

[9] Yusof, F. M., Ismail, A. I. M. and Ali, N. M. Modeling population harvesting of rodents for the control of Hantavirus infection. Sains Malaysiana. 2010. 39(6): 935-940.

[10] Rida, S. Z., Abdel Rady, A. S., Arafa, A. A.M. and Khalil, M. The effect of the environmental parameter on the Hantavirus infection through a fractional-order SI model. Int. J. Basic Appl. Sci. 2012. 1(2): 88-99.

[11] Yusof, F. M., Ismail, A. I. M. and Ali, N. M. Effect of predators on the spread of Hantavirus infection. Sains Malaysiana. 2014. 43(7): 1045-1051.

[12] Yusof, F. M., Abdullah, F. A. and Ismail, A. I. M. Modeling and optimal control on the spread of Hantavirus infection. Mathematics. 2019. 7, 1192: 1-11.

[13] Yusof, F. M., and Ismail, A. I. M. Modeling the transmission dynamics on the spread of Hantavirus Infection. Menemui Matematik (Discovering Mathematics). 2019. 41(2): 96111.

[14] Hale, B. M., and McCarthy, M. L. An Introduction to Population Ecology Harvesting a Population with Logistic Growth. J. 2005. [Online article]: cited on 31 Jan 2017, http://www.maa.org/press/periodicals/loci/joma/an-introduction-topopulation-ecology-harvesting-a-population-with-logistic-growth.

[15] Kar, T. K. and Pahari U. K. Modelling and analysis of a prey-predator system with stagestructure and harvesting. Nonlinear Anal. Real World Appl. 2007. 8(2): 601-609.

[16] Solomon, E. P., Berg, L. R. and Martin. D. W. Biology, International Student Edition. 7th edition. Thomson Learning, Inc., United States. 2005.

[17] Mills, N. J. Biodiversity loss and emerging infectious disease: An example from the rodentborne hemorrhagic fevers. Biodiversity. 2006. 7(1): 9-17.

[18] Peixotu, I. D. and Abramson, G. The effect of biodiversity on the Hantavirus epizootic. Ecology. 2006. 87(4): 873-879.

[19] Ermentrout, B. Simulating, analyzing, and animating dynamical systems: a guide to XPPAUT for researchers and students. SIAM. 2002.

[20] Gause, G. The struggle for existence. Dover Publications. Inc., New York, 1934.

[21] Mohd, M. H., Murray, R., Plank, M. J. and Godsoe, W. Effects of dispersal and stochasticity on the presence-absence of multiple species. Ecological Modelling. 2016. 342: 49-59.

[22] Mohd, M. H. Modelling the Presence-Absence of Multiple Species. Ph.D. Thesis. University of Canterbury, Christchurch, New Zealand. 2016. 